\title{
UTILIDAD DE LA REACCIÓN DE TUBERCULINA
}

\section{The usefulness of the tuberculin skin test}

\author{
Dr. Clemax Couto Sant'Anna \\ Profesor Asociado Departamento de Pediatría. \\ Facultad de Medicina, Universidade Federal do Rio de Janeiro (UFRJ) y Rede TB.
}

\begin{abstract}
THE USEFULNESS OF THE TUBERCULIN SKIN TEST
Tuberculin skin test (TST) is a test based on purified protein derivative of Mycobacterium tuberculosis (or PPD). TST has been considered a standard for identifying tuberculous infected people by the World Health Organization (WHO) since 1955. However, other immunological tests that measure the interferon gamma production (Interferon gamma release assays or IGRAs) in the individual's cells with latent tuberculosis (TB) infection (LTBI) have been used as surrogate TST, despite having certain restrictions. WHO has made several recommendations for preventive treatment in cases of LTBI aiming to supplant the universal shortage of PPD since 2014. Some rules for identifying LTBI individuals without the TST still reiterate the importance of this test in many countries.
\end{abstract}

Key words: tuberculin test, interferon- gamma release test, tuberculosis, child.

\section{RESUMEN}

La reacción tuberculina (RT) es una prueba cutánea que emplea el derivado proteico purificado de Mycobacterium tuberculosis o PPD. Desde 1955 ha sido considerada estándar para identificar personas infectadas por el bacilo de la tuberculosis por la Organización Mundial de la Salud (OMS). Sin embargo, otras pruebas inmunológicas que miden la producción de interferon gama (IGRAs) por las células del individuo con infección latente de TB (ILTB) se han empleado como sucedáneas de la RT, a pesar de presentar ciertas restricciones. La OMS ha hecho diversas recomendaciones para la conducta preventiva en casos de ILTB que de alguna forma buscan suplantar la falta universal de PPD desde 2014. Algunas perspectivas de identificar personas con ILTB sin contar con el PPD reiteran todavía la importancia de la RT en muchos países.

Palabras clave: prueba de tuberculina, ensayos de liberación de interferon gamma, tuberculosis, niños.

\section{HISTORIA DE LA REACCIÓN TUBERCULINA}

La prueba de tuberculina o reacción tuberculina (RT) es una prueba estándar para identificar personas infectadas por la tuberculosis por la Organización Mundial de la Salud (OMS) desde 1955. Se realiza en la mayoría de los países con el empleo del derivado proteico purificado (PPD) del Mycobacterium tuberculosis. Su principal productor es Statens Serum Institut (SSI) de Dinamarca el cual distribuyó el PPD-Rt 23. La prueba estándar es hecha con la inyección intradérmica de 2 unidades internacionales (UI) de tuberculina que corresponde a $0,1 \mathrm{ml}$, aplicada en la parte interna del antebrazo izquierdo y cuya lectura se realiza después de 48 a 72 horas. Se trata de una prueba inmunológica in vivo, la cual consiste en la intradermorreacción de tipo cuantitativo, descrita en 1910 por Mantoux, denominada reacción de Mantoux. EI PPD Rt-23 fue preparado a partir de un cultivo de siete muestras de Mycobacterium tuberculosis. Desde 1963 la OMS recomendó la estandarización de la reacción de Mantoux mediante el empleo del PPD Rt-23, debido a su potencia y estabilidad $(1,2)$. Otras técnicas han Ilegado a ser utilizadas, tales como la reacción de Von Pirquet por escarificación de la

\section{Correspondencia:}

Dr. Clemax Couto Sant'Anna

Dirección: Cinco de Julho 218 ap. 901 - Copacabana

Rio de Janeiro, RJ. Brasil

correo electrónico: clemax01@gmail.com piel, la oftalmo-reacción de Wolff-Eisner-Calmette, el patch-test 0 prueba de contacto, pistola y multipuntura o Tine- test.

La mayoría de los países adoptaron la reacción de Mantoux con PPD (o tuberculina), bajo la forma de prueba cutánea intradérmica de lectura tardía (en aproximadamente 72 h) con regla milimetrada. La reacción positiva a la prueba subentiende la medida - con regla milimetrada en el diámetro transverso del antebrazo - de la induración en la piel, de bordes firmes más 0 menos definidos, con al menos $6 \mathrm{~mm}$ con halo de eritema. Su aplicación masiva ha propiciado innumerables estudios epidemiológicos para el cálculo del riesgo de infección latente por TB (ILTB) en diversos países. La RT ayuda a diferenciar personas no infectadas de infectadas por TB, es decir, con ILTB, en las cuales correspondería la terapia preventiva de TB $(3,4)$.

La RT permite distinguir individuos reactivos $(>5 \mathrm{~mm})$ de no reactivos (0 a $5 \mathrm{~mm}$ ). Las reacciones superiores a $15 \mathrm{~mm}$ se consideran fuertemente positivas (o reactivas fuertes). Los reactivos a la $\mathrm{RT}$ se consideran infectados por el bacilo de la TB. La RT puede sufrir influencia de la vacunación BCG previa, por eso se debe tener cautela en su interpretación. Como la RT no es capaz de identificar personas infectadas específicamente por el M. tuberculosis, la infección por otras micobacterias, como el Mycobacterium bovis, BCG y micobacterias no tuberculosas (MNTB) puede influir en la reacción, llevando a un resultado falso positivo. Así, la interpretación de la RT debe tener en cuenta resultados falsos positivos y falsos negativos. Varias condiciones pueden ser anergizantes, como algunas virosis, particularmente 
el sarampión o enfermedades inmunosupresoras, y el embarazo. Es también importante evaluar la aplicación y lectura correctas de la RT en las unidades de salud (5). Las causas de resultados falso negativos están descritas en la Figura 1.

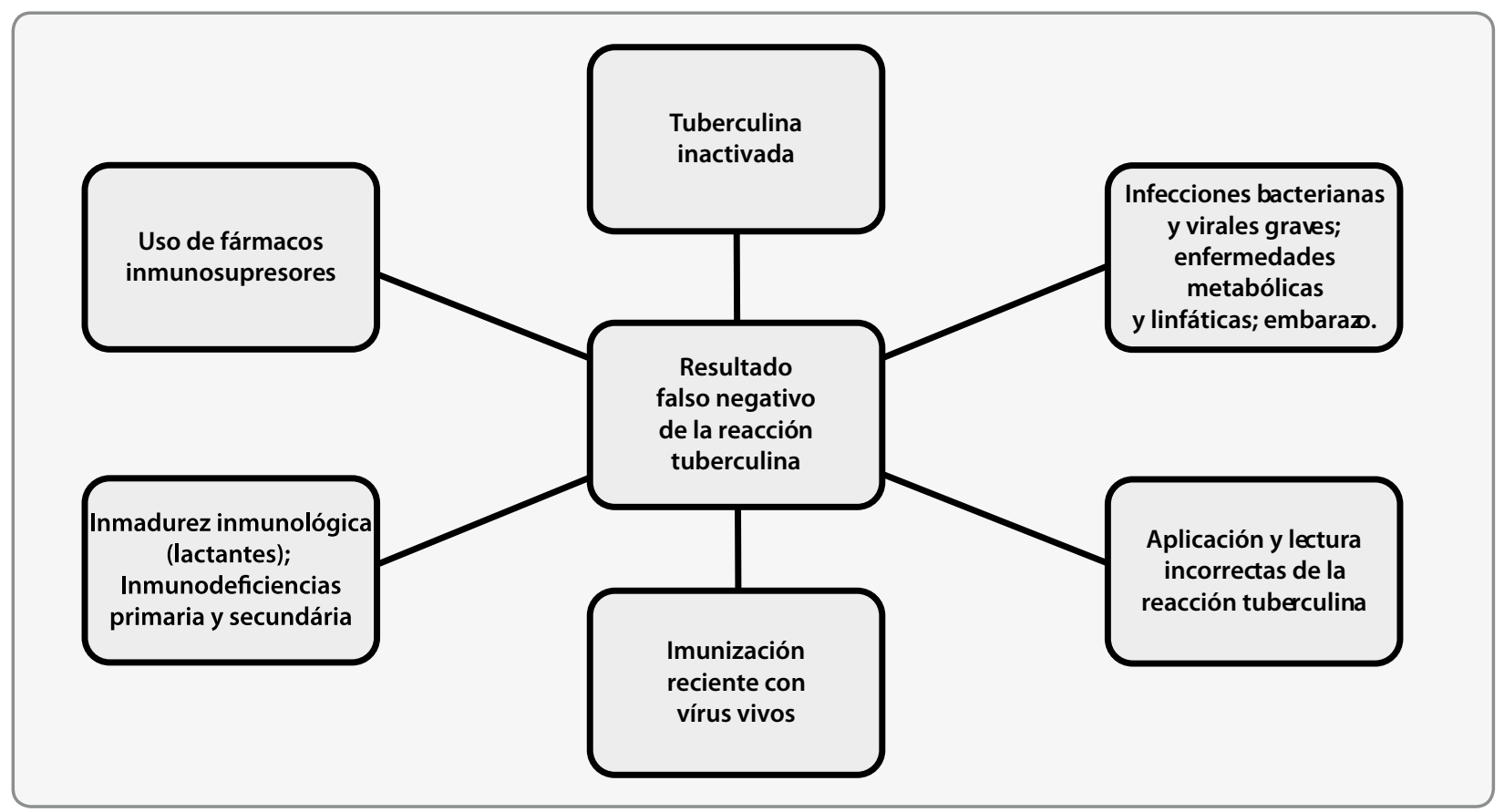

Figura 1. Causas de resultados falso-negativos de la prueba de la tuberculina.

\section{LA REACCIÓN TUBERCULINA SERIADA}

Cuando el resultado de la RT es no reactivo se puede proceder a la repetición de la prueba o la RT seriada. Hay dos fenómenos que se deben considerar cuando se realiza la RT seriada 0 en dos etapas: el efecto booster y la conversión reciente 0 el viraje de la tuberculina.

La conversión o viraje de la tuberculina puede ocurrir cuando se realiza la RT en dos etapas. Esto es, cuando, en la evaluación inicial de un contacto la $\mathrm{RT}$ es no reactiva $(<5 \mathrm{~mm})$ y al ser repetida 8 a 10 semanas después hay incremento $\geq$ $10 \mathrm{~mm}$. Este fenómeno indica que el individuo estaba en una fase anérgica. Es decir, tenía ILTB pero todavía no había trascurrido tiempo suficiente para que hubiera exteriorización demostrada por la reacción cutánea. Por eso la prueba inicialmente era falsamente negativa. Así, la segunda prueba reveló que la persona estaba de hecho infectada.

Otro fenómeno es el efecto booster que es poco común en la infancia. Puede ocurrir también en la prueba seriada con la RT. El efecto booster se manifiesta en individuos con exposición remota al $M$. tuberculosis o a MNTB, en general en adultos con más de 55 años y en personas vacunadas previamente (después de la primera infancia) con BCG. De esta forma, la reactividad observada en la segunda prueba en estos individuos refleja la infección previa por TB que no se había manifestado en la primera RT. Si la segunda RT fuese no reactiva, sería prácticamente cierto que la persona no estaba infectada por la TB. Los no infectados no reaccionan a la RT seriada. Estos individuos se deben controlar periódicamente para identificar posibles virajes de la tuberculina a lo largo del tiempo, según acciones de control de TB, adotadas por algunos países $(3,6)$.

\section{LA INFLUENCIA DE LA VACUNACIÓN BCG SOBRE LA REACCIÓN TUBERCULINA}

El efecto de la vacunación BCG sobre la reacción tuberculina es conocido pero es todavía difícil de cuantificar, pues depende de diferentes cepas de vacuna y de características peculiares de las poblaciones estudiadas, así como de diferencias en la producción de la tuberculina empleada en las pruebas. Muchos autores y guías terminan arbitrando valores de puntos de corte para la reacción tuberculina en niños vacunados con $B C G$. De todo modo, es cierto que la reacción de la tuberculina disminuye con la edad en los niños vacunadas al nacer y que el diagnóstico de ILTB no es fácil en niños vacunados con BCG. Chan et al (7) en Twain, país de mediana prevalencia de $T B$, identificaron que la reacción de la tuberculina disminuye hasta los 7 años y de ahí en adelante volvía a elevarse. Una de las explicaciones para este hallazgo es que las infecciones por MNTB podrían justificar dicho fenómeno en una región subtropical, como aquella. Trabajos más antiguos, en los que observaron la disminución y aumento de la RT con la edad de los niños probados, identificaron puntos de corte diferentes según 
las poblaciones estudiadas. Así, países africanos de elevada prevalencia de TB o niños de áreas pobres en Río de Janeiro, Brasil, exhibían disminución de la reacción de la tuberculina con el tiempo pero como consecuencia de nuevas exposiciones a la TB en aquellas áreas, volvían a reaccionar a la RT (8).

La RT es útil hasta en niños vacunados con BCG. Se puede considerar reactiva si $\geq 5 \mathrm{~mm}$ y no reactiva si inferior a $5 \mathrm{~mm}$. Cuando es reactiva permite identificar la infección por $M$. tuberculosis, es decir, sirve para informar sobre probable ILTB. En Brasil en niños vacunados con BGC desde hace menos de 2 años, se considera la RT sugestiva de infección por $M$. tuberculosis cuando la reacción sea $\geq 10 \mathrm{~mm}$. A partir de 2 años de la vacunación BCG, la RT se considera reactiva si $\geq 5 \mathrm{~mm}$ (9).

\section{LOS IGRAS Y LA PRUEBA DE LA TUBERCULINA}

Las pruebas in vitro que miden la producción de interferon gama, denominados Interferon-Gamma Release Assays (IGRAs) usan antígenos específicos del M. tuberculosis, como early secretory antigenic target 6 (ESAT-6), culture filtrate protein 10 (CFP-10) y TB 7.7. Los genes que codifican estos antígenos son encontrados en $M$. Tuberculosis pero no en $M$. bovis. Por eso no conducen a resultados falsos positivos en niños vacunados con BCG. Se dispone de dos pruebas rápidas para diagnóstico de ILTB para uso comercial. Cuantiferon (CTF)TB Gold, basado en el método ELISA para medir la producción del interferon gama (IFN y) de las células $T$ en la sangre total y T-SPOT.TB que usa la técnica del ELISPOT para medir esa producción de IFN y (5).

La reacción tuberculina y Ios IGRAs son pruebas inmunológicas indirectas, que detectan la respuesta inmune celular a la sensibilización reciente o pasada a antígenos de micobacterias. No sirven para distinguir ILTB de la enfermedad actual (TB activa) o pasada. En 2011 dos metanálisis refirieron que en niños la RT y los IGRAs poseen precisión semejante para detección de ILTB o TB activa. Un metanálisis reciente muestra que el comportamiento de los IGRAs en pacientes infectados y no infectados por el VIH con ILTB fue idéntico al de la RT. Los autores afirman que la adopción de uno u otro, cuyos resultados son modestos en la ILTB, depende de cada país $(5,10,11)$.

\section{LA FALTA DE PPD Y ALTERNATIVAS}

En un documento de 2014 la OMS cita la RT como elemento fundamental para el diagnostico de la TB en la infancia, además de otros datos (12). La falta de PPD alcanzó el mundo a partir de 2014 y provocó algunas manifestaciones expresivas de la comunidad médica. Esta falta de materia prima se debió al cese de distribución del PPD por el SSI en Dinamarca. El
SSI fue privatizado y los cambios administrativos lo llevaron a interrumpir el suministro del producto.

El Grupo de Ensayos de la Red Europea de Tuberculosis Pediátrica manifestó la falta de información sobre la escasez de PPD y la necesidad de superarla, una vez que el PPD se considero esencial para el mantenimiento de la RT como recurso crucial en términos de salud pública. Treinta y cinco médicos de 23 países europeos fueron consultados sobre la falta de PPD, estos refirieron proceder a la limitación de las existencias remanentes para solo en casos especiales y comentaron sobre la sustitución de esta prueba por los IGRAS, aunque éstos tengan baja sensibilidad en niños pequeños y sean de costo elevado. La Red europea llegó a proponer la necesidad de una agencia supranacional con el objetivo de monitorear la producción de PPD para asegurar la disponibilidad del producto en virtud de su importancia en niños (13).

La sustitución de la RT por los IGRAS tiene innumerables limitaciones, y llegó a no ser recomendada en áreas donde la TB es endémica. Las principales desventajas de Ios IGRAs son: elevado costo y técnica más compleja en comparación con la RT y toma de muestra de sangre de los individuos para la prueba. En la mayoría de los países con recursos limitados, y en general, en áreas de elevada endemia de TB (donde la incidencia es $\geq$ 100/100 00 hab, según la OMS), no se recomiendan por las directrices para uso en salud pública en sustitución a la RT, principalmente en pacientes inmunosuprimidos $(10,14,15)$.

Por otro lado, Mandalakas et al (16) destacan el valor de IOS IGRAS en niños jóvenes infectados por VIH para la detección de ILTB en país con elevada prevalencia de TB, como es el caso de la África del Sur. No obstante, hasta el momento, la sustitución de la RT por Ios IGRAS, no se recomienda en niños pequeños, pues presenta algunos inconvenientes. La precisión y valor predictivo de los IGRAs son idénticos a los de la RT en el sentido de identificar ILTB, a pesar de que la literatura ha reportado que en los países donde la TB es endémica hay muchos resultados discordantes entre estos métodos. El Ministerio de Salud de Brasil estableció la comparación entre estos dos métodos apuntando sus ventajas y desventajas (Tabla 1) 


\begin{tabular}{|c|c|}
\hline PRUEBA TUBERCULINA & IGRAs \\
\hline Entrenamiento difícil & Entrenamiento difícil \\
\hline $\begin{array}{l}\text { Se puede usar en forma seriada } \\
\text { (se puede usar en profesionales de salud, } \\
\text { personas que viven con VIH, contactos) }\end{array}$ & $\begin{array}{l}\text { Pruebas seriadas no confiables } \\
\text { (reversión y conversión espontáneas) } \\
\text { no indicado en profesionales de salud, } \\
\text { personas que viven con VIH y contactos }\end{array}$ \\
\hline $\begin{array}{l}\text { Evidencias de alta calidad del beneficio } \\
\text { del tratamiento de la ILTB en aquellos } \\
\text { que tienen reacción tuberculina positiva }\end{array}$ & Apenas inferencia \\
\hline $\begin{array}{l}\text { Necesidad de retorno para lectura } \\
\text { del examen implica en muchas pérdidas }\end{array}$ & Diagnóstico obtenido en visita única \\
\hline Prescinde del laboratorio & Necesita laboratorio \\
\hline Posibilidad de efecto booster & Efecto booster apenas si reacción tuberculina anterior \\
\hline No hay resultado indeterminado & $\begin{array}{l}\text { Tasa de resultados indeterminados (descrita como 2\%); } \\
\text { se puede elevar en algunas series }\end{array}$ \\
\hline
\end{tabular}

Tabla 1. Comparación entre la reacción tuberculina y los métodos de producción de interferon gama (IGRAs).

\section{PERSPECTIVAS}

En 2015 la OMS estableció normas para la conducta en casos de ILTB frente a la nueva realidad - la falta de PPD - buscando normalizar las acciones preventivas contra la TB en países de elevada y mediana tasa. El primer aspecto es la búsqueda de casos de TB entre los contactos investigados. En este sentido toda investigación se debe iniciar por la búsqueda de señales y síntomas sugestivos de TB entre los contactos, antes de probarlos en cuanto a la posibilidad de ILTB. El empleo de la radiografía de tórax debe considerarse en los individuos sintomáticos y en el diagnóstico diferencial con otras enfermedades. La RT y los IGRAs se pueden usar en la identificación de casos de ILTB en países cuya incidencia de TB sea inferior a 100/100.000 hab. Según la OMS estas recomendaciones recibieron la clasificación: recomendación fuerte; nivel muy bajo de evidencia (15).

En Brasil, debido a la falta momentánea de PPD, el Ministerio de Salud propuso el abordaje de los contactos sin la prueba de la tuberculina, como se muestra en la Tabla 2.

\begin{tabular}{|c|c|}
\hline GRUPOS POR EDAD & CONDUCTA \\
\hline Niños, adolescentes y adultos sintomáticos & Investigar TB en actividad \\
\hline Niños $\leq 5$ años asintomáticos & $\begin{array}{c}\text { Excluir TB en actividad; tratar la ILTB } \\
\text { sin la prueba de la tuberculina }\end{array}$ \\
\hline Niños > 5 años asintomáticos & $\begin{array}{l}\text { Excluir TB y considerar individualmente indicación de } \\
\text { tratamiento de la ILTB sin la prueba de la tuberculina }\end{array}$ \\
\hline Adultos y adolescentes asintomáticos & $\begin{array}{c}\text { Tener en cuenta: riesgo/beneficio, grado de exposición } \\
\text { y comorbilidades para tratar la ILTB }\end{array}$ \\
\hline
\end{tabular}

Tabla 1. Comparación entre la reacción tuberculina y los métodos de producción de interferon gama (IGRAs). 
Finalmente, cabe recordar la posibilidad de desarrollo de un PPD recombinante, es decir, el empleo de proteínas recombinantes del PPD que no estarían presentes en la vacuna BCG y que, por lo tanto, harían la prueba más específica para identificar la ILTB. Por ahora, el PPD recombinante se encuentra en nivel experimental, principalmente en bovinos, pero su utilización en humanos es muy prometedora y sería una nueva perspectiva, 0 una nueva fase, del empleo de la RT en el mundo $(17,18)$.

\section{El autor declara no presentar conflicto de intereses}

\section{AGRADECIMIENTOS.}

A los Doctores María de Fátima B. Pombo March y Andrés Zorrilla Vaca por las contribuciones sustantivas al artículo.

\section{REFERENCIAS}

1. Magnusson $M$, Bentzon MW. Preparation of purified tuberculin RT-23. Bull WHO 1958; 19: 829-43

2. World Health Organization. The WHO standard tuberculin test. WHO/TB/ Technical Guide/ 3.22 February 1963

3. Bethlem N. Pneumologia. Atheneu, Rio de Janeiro, Brasil, 1973 p 185-226

4. Sant'Anna CC, Loboguerrero MA, Pequeno RF. Profilaxis con isoniazida en niños y jóvenes con infección latente por Mycobacterium tuberculosis. Salud(i)Ciencia 2014; 20: 630635

5. Perez-Velez CM, Marais BJ. Tuberculosis in children. N Engl J Med. 2012; 367:348-61

6. Teixeira EG, Kritski A, Ruffino-Netto A, Steffen R, Lapa e Silva JR, Belo M Luiz RR, Menzies D, Trajman A. Two-step tuberculin skin test and booster phenomenon prevalence among Brazilian medical students. Int J Tuberc Lung Dis 2008;12:1407-1413

7. Chan P-C, Chang L-Y, Wu Y-C, C-Yi Lu, C-Yi, Kuo H-S, Lee C-Y, Huang LM, Chen CJ. Age-specific cut-offs for the tuberculin skin test to detect latent tuberculosis in BCG-vaccinated children. Int J Tuberc Lung Dis 2008;12:1401-1406

8. Hijjar MA. Aspectos do controle da tuberculose numa população favelada: favela do Escondidinho, Rio de Janeiro. Tesis. Faculdade de Medicina. Departamento de Doenças Infecciosas e Parasitarias 1985.

9. Brasil. Ministério da Saúde. Manual de recomendações para o controle da tuberculose. Brasília, 2010. Disponível em: http://www.cve.saude.sp.gov.br/htm/TB/mat_tec/manuais/ MS11_Manual_Recom.pdf

10. Sztajnbok FR, Boechat NL, Sztajnbok DCN, Brumibeiro S, Oliveira SK, Sant'Anna CC. The challenge of pediatric tuberculosis in face of new diagnostic techniques. J. Ped (Rio J) 2009; 85:183-93
11. Cattamanchi A, Smith R, Steingart KR, Metcalfe JZ, Date $A$, Courtney $C$ et al. Interferon-gamma release assays for the diagnosis of latent tuberculosis infection in HIVinfected individuals - A systematic review and metaanalysis. JAIDS. Publish Ahead of Print DOI: 10.1097/ QAl.0b013e31820b07ab

12. WHO. Guidance for national tuberculosis programmes on the management of tuberculosis in children. 2 a. ed, Geneva, 2014. p126

13. Tebruegge $M$, Bogyi $M$, Soriano-Arandes $A$, Kampmann $B$ on behalf of the Paediatric Tuberculosis Network. European Trials Group. Shortage of purified protein derivative for tuberculosis testing. Lancet 2014; 384: 2026

14. Getahun H, Sculier D, Sismanidis C, Grzemska M, Raviglione M. Prevention, diagnosis and treatment of tuberculosis in children and mothers: evidence for action for maternal, neonatal, and child Health Services. JID 2012; 205: S216227

15. WHO. Guidelines on the management of latent tuberculosis infection. The End TB Strategy. Geneva, 2015, p 33

16. Mandalakas AM, Kirchner HL, Walzl G, Gie RP, Schaaf HS, Cotton MF et al. Optimizing the detection of recent tuberculosis infection in children in a high tuberculosis-HIV burden setting. AJRCCM 2015; 191: 820-830

17. Borsuk S, Newcombe J, Mendum TA, Dellagostin OA McFadden J. Identification of proteins from tuberculin purified protein derivative (PPD) by LC-MS/MS. Tuberculosis 2009; 89: 423-430

18. Malaghini M. Proteinas recombinates de M. tuberculosisi prap auxilio daignóstico da tuebrculose. Tesis. Pós-Graduação em Processos Biotecnológicos. Universidade Fedral do Paraná Curitiba, 2008. p158 